Musées, Patrimoine et Culture scientifiques et techniques

$159 \mid 2015$

mai-juin 2015

\title{
La mise en place des politiques publiques au Jardin botanique de Nantes
}

\section{Romaric Perrocheau}

\section{OpenEdition \\ Journals}

Édition électronique

URL : https://journals.openedition.org/ocim/1518

DOI : 10.4000/ocim.1518

ISSN : 2108-646X

Éditeur

OCIM

Édition imprimée

Date de publication : 1 mai 2015

Pagination : 26-29

ISSN : 0994-1908

Référence électronique

Romaric Perrocheau, «La mise en place des politiques publiques au Jardin botanique de Nantes », La Lettre de l'OCIM [En ligne], 159 | 2015, mis en ligne le 01 mars 2016, consulté le 21 septembre 2021. URL : http://journals.openedition.org/ocim/1518; DOI : https://doi.org/10.4000/ocim.1518

Ce document a été généré automatiquement le 21 septembre 2021.

Tous droits réservés 


\section{La mise en place des politiques publiques au Jardin botanique de Nantes}

\section{Romaric Perrocheau}

Les serres du Jardin botanique de Nantes.

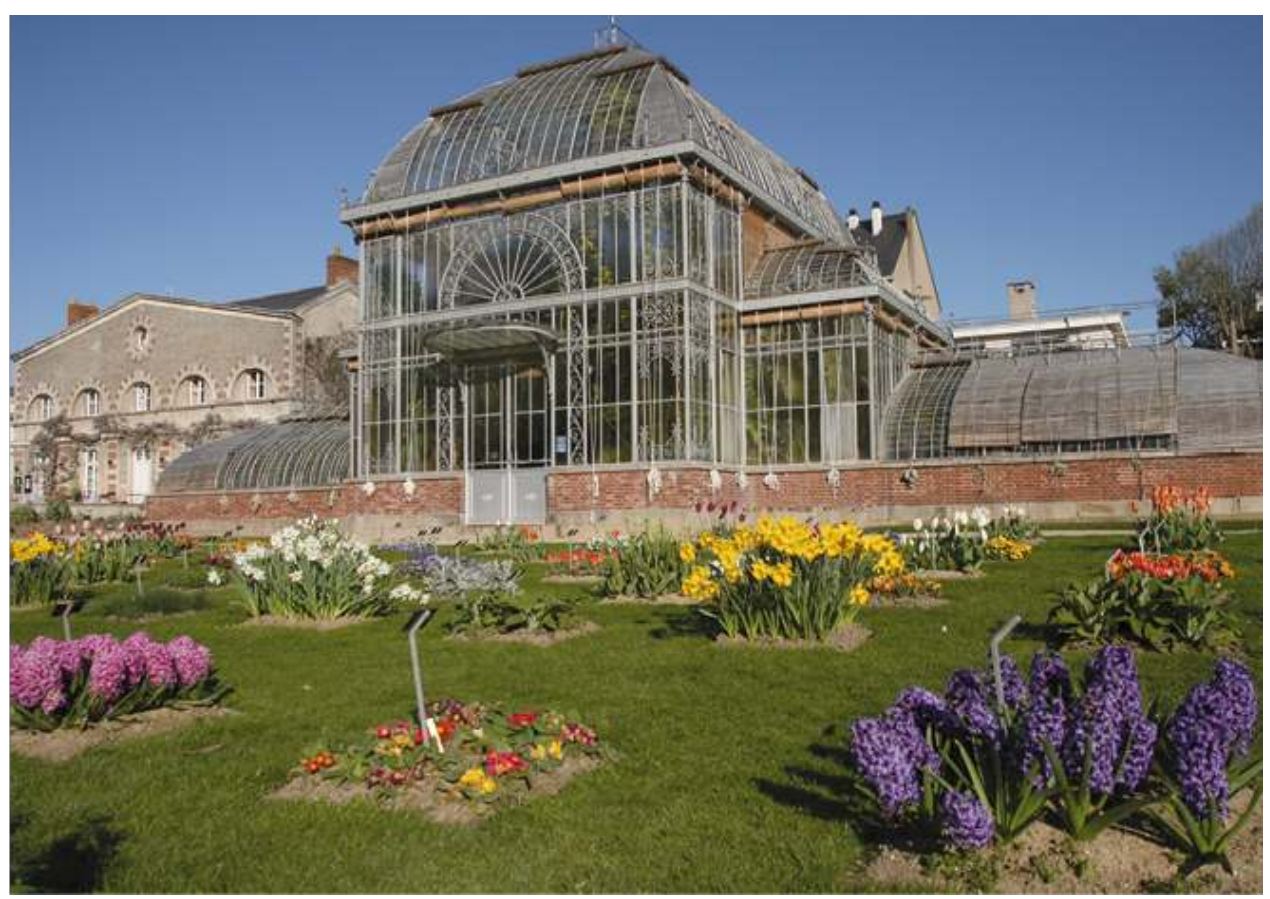

(c) Jardin botanique de Nantes 


\section{Des expertises croisées au sein de la municipalité}

1 La ville de Nantes possède deux institutions scientifiques complémentaires : le muséum d'Histoire naturelle et le Jardin botanique, chaque entité a un champ d'expertise spécifique, la faune et la géologie pour le premier et la flore pour le second.

2 Environ 40 agents travaillent au muséum, qui accueille 70000 visiteurs par an et qui dépend de la Direction de la Culture, tandis que 25 personnes travaillent au jardin botanique, qui accueille près de 2 millions de visiteurs annuels et qui dépend du Service des Espaces Verts et de l'Environnement (500 agents).

3 Les collaborations entre ces deux institutions sont riches et fréquentes dans tous leurs domaines de compétence: inventaires entomologiques au Jardin botanique par les équipes du muséum, détermination et prélèvement de végétaux par les agents du Jardin botanique dans le cadre d'expositions au muséum, conférences à deux voix...

4 Au-delà de ces champs classiques de collaboration, les deux établissements sont les référents de la ville dans le cadre d'une structure originale: le Conseil Nantais de la Biodiversité. Il s'agit d'une structure locale, voulue par le maire qui regroupe experts nationaux, associations naturalistes locales et services de la ville afin d'émettre des avis et des recommandations pratiques sur les politiques publiques menées par la ville en matière de biodiversité.

Session de formation aux inventaires participatifs de papillons avec les jardiniers référents biodiversité.

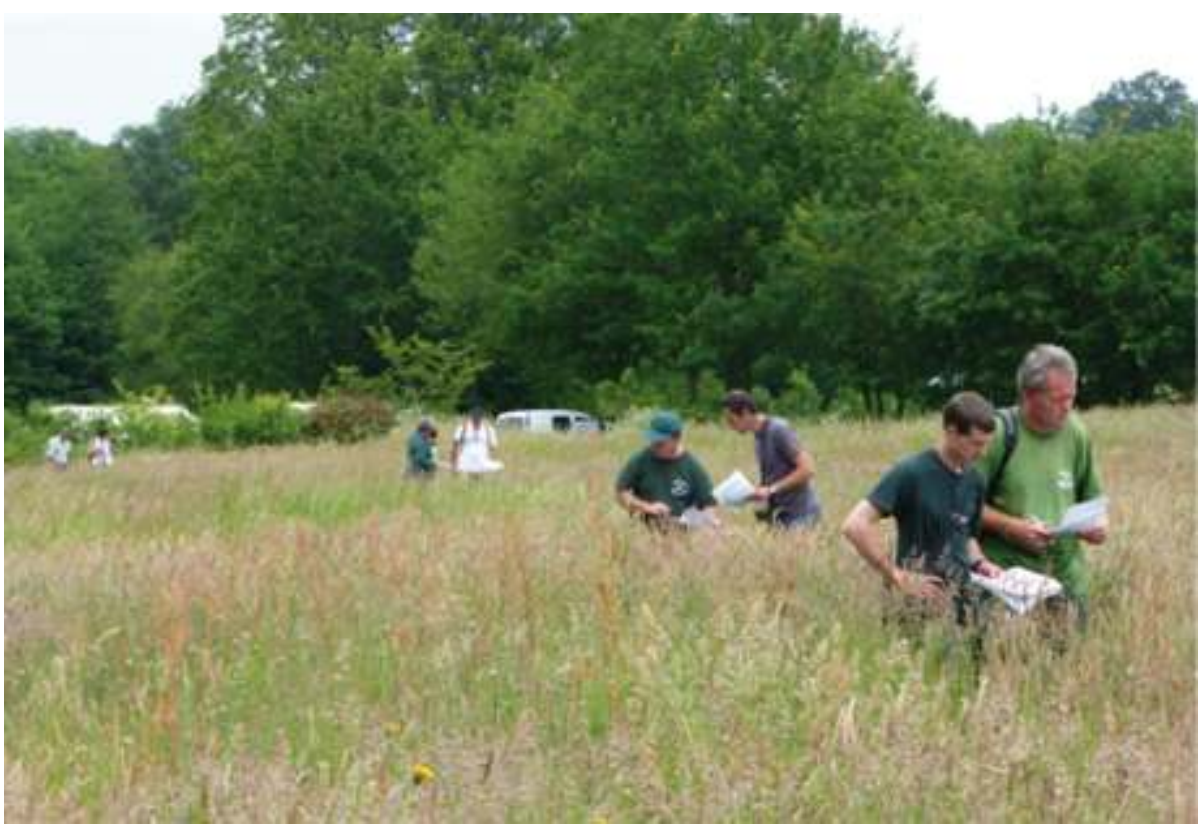

(C) Jardin botanique de Nantes

\section{Des expertises menées " hors champs»}

La culture de l'intégralité des espèces constituant la flore du massif armoricain (1500 taxons) depuis plus de 100 ans a enrichi le Jardin botanique d'un savoir-faire dépassant le 
simple savoir théorique et reconnu par tous, ce qui permet de mener des expérimentations sur le vivant. Prenons deux exemples bien différents.

Depuis plus de 50 ans, le Jardin botanique cultive dans une parcelle de vigne expérimentale 37 bulbes (devenus 2000 avec le temps) de tulipe sylvestre, une espèce disparue depuis lors dans le département et bénéficiant d'un plan de protection national. Les conditions de culture, étudiées chaque année, ont entraîné le dépôt d'un dossier de réintroduction auprès du Conseil National de Protection de la Nature, et la réunion de celles-ci chez deux viticulteurs bio en muscadet.

7 Un partenariat au long cours avec les allergologues a permis de développer un système de détection des premières émissions de pollen complémentaire aux capteurs polliniques «classiques ». Ce pollinarium, constitué des 20 espèces les plus allergisantes de la région et observé chaque jour par un jardinier botaniste qui déclenche les alertes auprès de l'Agence Régionale de Santé a rencontré un tel succès qu'il essaime maintenant dans de nombreuses villes de France.

\section{La participation}

8 L'expertise de haut niveau et durant une longue période n'a d'intérêt pour la Cité que si elle est partagée. Là encore, deux exemples peuvent éclairer cet axe.

9 Le Jardin botanique propose un « cours municipal de botanique » qui dure deux années et qui est proposé à tous les Nantais. Ce cours de 80 heures reprend tous les fondamentaux de la botanique (anatomie, physiologie, reproduction...). Il est constitué de cours bimensuels en salle durant lesquels une vingtaine d'échantillons prélevés dans le Jardin botanique sont présentés. Il est complété par plusieurs sorties sur le terrain.

10 L'association Botanica qui en est issue est accompagnée par le Jardin botanique pour réaliser des inventaires participatifs sur les trottoirs type «Sauvages de ma rue ». Ceux-ci sont les plus anciens réalisés avec régularité en France.

11 Le Jardin botanique pilote «Les référents biodiversité » au sein du Service des Espaces Verts. Il s'agit d'un groupe d'une quarantaine de personnes constitué d'un à deux agents par équipe sensible aux questions écologiques. Ces agents suivent des formations thématiques pointues, trois journées par an, balayant des sujets relatifs aux milieux (zones humides, boisements...) ou aux espèces (chauve-souris, vers de terre...) par les spécialistes nationaux (par exemple du Muséum national d'Histoire naturelle). Après plusieurs années, ces agents acquièrent un réel savoir et un savoir-faire qu'ils diffusent au sein de leur équipe. Ils deviennent également des relais de qualité pour mener des inventaires participatifs sur leur territoire, comme PROPAGE, inventaire relatif aux papillons ou l'OPVT relatif aux vers de terre. Ils peuvent ensuite analyser les évolutions sur les habitats qu'ils gèrent sans attendre des retours extérieurs de bureau d'études, en constatant directement des modifications dans les populations suivies.

\section{La culture}

12 Là encore, deux exemples peuvent illustrer le positionnement des jardins botaniques du $\mathrm{XXI}^{\mathrm{e}}$ siècle bien au-delà des frontières physiques du jardin dans le cadre de collaborations artistiques. 

des plantes, mais équipée de dirigeables gonflables pour s'envoler et de pattes métalliques géantes pour l'atterrissage a été conçue en collaboration entre le Service des Espaces Verts de la Ville de Nantes et la compagnie La Machine. Cette étrange structure de plus de 12 mètres de haut a déjà été observée à Hambourg, Turin, Santiago du Chili ou Buenos Aires. Ces expéditions des temps modernes ont pour but de découvrir et de décrire la flore observée dans les villes étapes afin d'en détecter le potentiel photoélectrique. L'équipage est constitué de membres de la compagnie La Machine et de botanistes du Jardin botanique.

Atterrissage de l'Aéroflorale lors de l'expédition « Capitale verte Européenne à Nantes ».

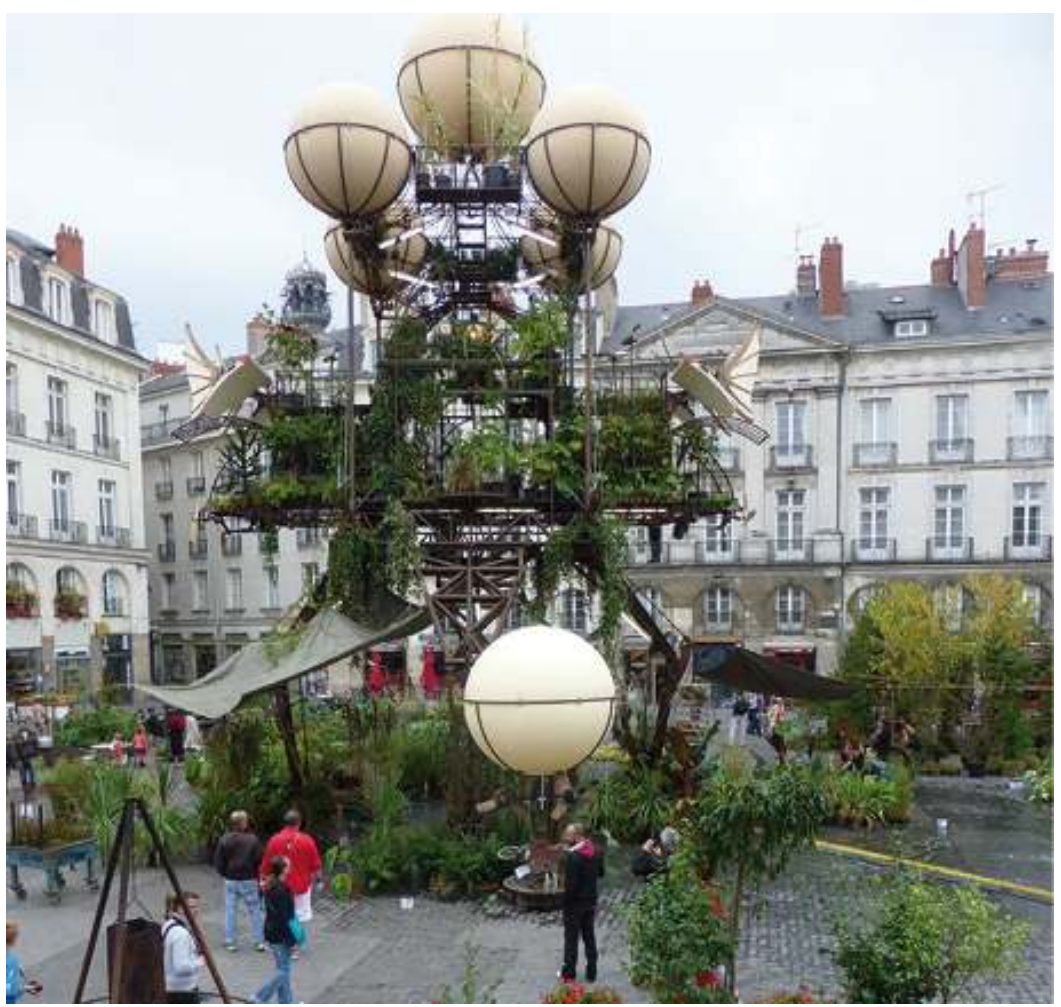

(C) Jardin botanique de Nantes

Cette même compagnie a pour objectif de bâtir un arbre de métal de 35 mètres de haut, avec $2 \mathrm{~km}$ de promenades intérieures, survolé par deux hérons pouvant transporter des visiteurs. Une première branche prototype est en place depuis 8 ans et pratiquée chaque jour par des milliers de visiteurs. La sélection des espèces, le recueil des graines, la mise en culture, la plantation et le suivi scientifique de l'adaptation de ces plantes chasmophytes à cet environnement spécifique sont réalisés par les équipes du Jardin botanique avec comme objectif de pouvoir les décliner demain à grande échelle.

Par ailleurs chaque année, le jardin accueille un artiste qui revisite ses plantations annuelles (50 000 plantes annuelles implantées chaque année). Après la venue de l'artiste Kynia Maruyama le Jardin botanique accueille pour la troisième année consécutive l'auteur d'albums pour enfants le plus connu en France, Claude Ponti, qui imagine un mobilier onirique, des animaux-végétaux qui valorisent le savoir-faire horticole des jardiniers tout en le rendant très séduisant auprès du grand public. 


\section{Le tourisme et la mise en réseau des jardins à l'échelle de la ville}

jardins. Cependant, tous n'ont bien évidemment pas le même intérêt touristique. C'est pourquoi 10 d'entre eux ont été retenus pour être mis en avant afin de montrer qu'il existe une réelle destination "jardins " à Nantes. Ceux-ci ont été retenus pour leur surface ( 2 ha minimum par jardin) et leur complémentarité thématique : jardin japonais, jardin romantique du XIX ${ }^{\mathrm{e}}$ siècle, roseraie, arboretum...

Un travail d'information par une signalétique sur place, des dépliants, un site Internet... a contribué à construire une image unique et un logo spécifique "Parcs et Jardins de Nantes ».

Après avoir bâti ce produit « Parcs et Jardins de Nantes » bénéficiant d'une image unique de qualité, nous avons pu nous intégrer dans le dispositif plus global de la mise en tourisme de la ville de Nantes et de ses environs. En effet, Jean-Marc Ayrault a souhaité, en tant que maire de Nantes, faire fusionner les différents équipements touristiques (les machines de l'île et son célèbre éléphant géant, le château des Ducs de Bretagne, la biennale d'art contemporain Estuaires et les offices de tourisme de l'agglomération) dans le but de proposer une offre cohérente aux visiteurs.

Cette structure baptisée « Le Voyage à Nantes » et présidée par Jean Blaise a rapidement joué le rôle de chef d'orchestre de l'ensemble des structures, privées comme publiques, qui accueillent des touristes. La création de cette entité tombait à point nommé pour intégrer le Jardin botanique dans une démarche plus globale et promouvoir son image dans les autres villes de France et à l'étranger.

Cette mise en réseau tout au long de l'année se décline aussi lors des nombreux rendezvous éphémères que sont les festivals. C'est ainsi que le Jardin botanique a pu s'associer au festival SPOT (rencontres de jeunes artistes locaux), cinéma plein air, heures d'été (concerts et lectures publiques dans toute la ville), SCOPITONE (festival de musiques électroniques)... qui permettent à la fois de bénéficier d'une très forte visibilité et d'attirer un public peu habitué à venir au Jardin botanique.

\section{La fréquentation du Jardin botanique de Nantes}

Le Jardin botanique accueille plus de 10000 taxons, le plaçant dans les 5 premières collections nationales. Une partie de ces collections est cultivée dans des serres ouvragées du XIXe siècle. Aussi, durant 10 ans un travail de fond a été réalisé afin de réhabiliter chacune des 10 serres et compléter les collections botaniques pour proposer chaque année un secteur dédié à la botanique totalement remis à jour.

Il est ainsi possible de découvrir le jardin en 30 minutes (visite libre des serres avec code d'accès retiré au bureau d'accueil) ou en 2 ans (cours municipal de botanique), que l'on soit enfant (atelier scolaire) ou adulte (visite nocturne des serres), en autonomie (jeu de piste avec manipulations) ou accompagné (800 visites guidées gratuites par an), seul ou en groupe (prestations spécifiques pour les groupes constitués). 

tout premier lieu l'écosystème urbain. Le rôle des citoyens et notamment leur participation dans l'ensemble des domaines de compétence des jardins botaniques sera certainement une des clés de leur rôle dans la cité de demain.

Le Jardin botanique, un lieu de ressource

Pourquoi les musées d'Histoire naturelle et les jardins botaniques ont-ils un rôle à jouer qui dépasse largement l'accumulation indispensable des connaissances? Pourquoi les communes doivent-elles s'inscrire à part entière dans cette recherche ? Comment celle-ci peut être non seulement reconnue dans les politiques publiques mais aussi être un point d'appui sur lequel construire le développement d'une ville dans laquelle les habitants se sentent partie prenante à part entière? 
Séance de détermination de plantes pendant un cours de botanique.

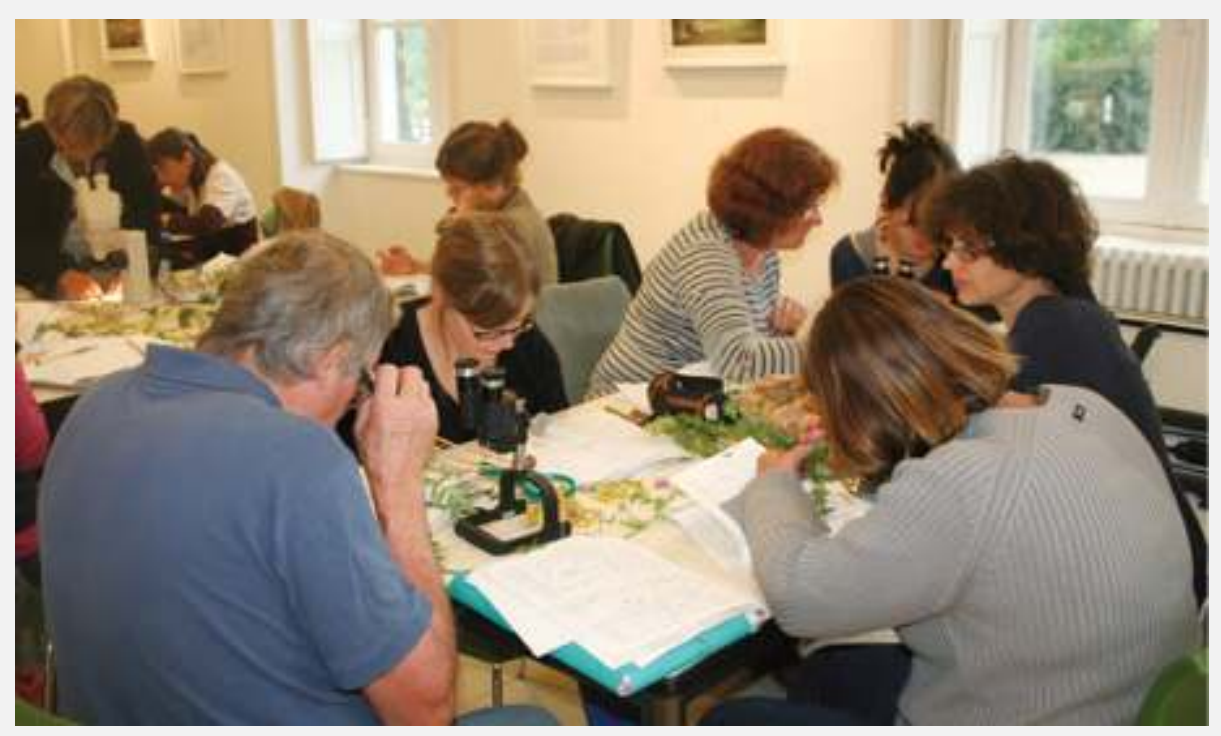

(C) Jardin botanique de Nantes

L'approfondissement des connaissances dans ces domaines où nous voyons qu'il y a encore tant à découvrir est essentiel. Mais cela ne peut être poursuivi et porté que si cela ne semble pas la spécialité de quelques-uns travaillant en cercle fermé. Les savoirs et recherches peuvent déjà être une ressource précieuse en interne : le Jardin botanique joue un rôle déterminant dans l'animation du Conseil Nantais de la Biodiversité. Mais c'est aussi une ressource pour des partenaires extérieurs dans le domaine culturel, ceux de l'urbanisme, de la santé ou de l'agriculture : le Jardin botanique participe aux essais pour végétaliser une branche de l'arbre aux hérons, végétaliser une nouvelle école en construction ou pour réintroduire la tulipe sylvestre dans les vignes de la région nantaise... Enfin le Jardin botanique est un acteur du développement d'expertises partagées avec par exemple des inventaires participatifs.

Le développement des connaissances nous concerne tous : que l'on soit néophyte ou spécialiste, s'intéresser au monde qui nous entoure, apprendre à connaître les mécanismes et les interactions est un enrichissement pour chaque individu comme pour la vie collective. Mais cela ne se fait pas spontanément. Il faut susciter la question, donner l'envie, et donc organiser les services et lieux pour qu'ils soient une véritable porte d'entrée à ce questionnement. Chacun doit se sentir accueilli quelles que soient ses connaissances préalables. L'organisation des services comme celui des lieux doit donc intégrer cet objectif.

Le Jardin botanique organise de nombreux ateliers scolaires adaptés aux différents âges. Et ceux qui désirent approfondir leurs connaissances peuvent s'inscrire aux ateliers botaniques. Il s'agit donc de mettre en avant une démarche : privilégier la diversité des approches afin de permettre au plus grand nombre de trouver un intérêt à fréquenter ces lieux et à développer ses connaissances. 
À Nantes, le Jardin botanique est devenu le point de départ ou point d'accueil d'évènements qui contribuent à l'attractivité de la ville et permettent aux habitants de se sentir parties prenantes de moments valorisants de la vie de la ville. Ces évènements sont des leviers pour mobiliser d'autres politiques publiques, ils croisent des politiques culturelles, éducatives, sociales et ainsi contribuent à renforcer le dynamisme de la ville et la qualité de vie pour tous les habitants. Ainsi, une collaboration avec Claude Ponti, auteur de livres pour enfants, invite les visiteurs à entrer dans son univers magique de dessinateur et à découvrir des créations « vertes » dans tout le parc. De nombreux visiteurs apportent une petite pierre à la création de cet univers : les enfants d'un centre de loisirs, les scolaires mais aussi des artisans et des personnes âgées qui dans les quartiers au cours de l'année ont participé à des ateliers pour créer des accessoires que l'on va retrouver dans le parc. Ainsi il ne s'agit pas seulement d'un évènement que l'on va voir mais c'est aussi une action à laquelle on a participé, à laquelle on a plaisir à inviter ses amis. Tout le travail du Jardin botanique est ainsi valorisé. La cohésion sociale, la qualité de la vie dans la ville se trouvent renforcées.

Catherine Touchefeu

Adjointe au Maire de Nantes, quartier Doulon/Bottière, déléguée Parcs, Jardins, Espaces verts (2008-2014)

\section{RÉSUMÉS}

Expert reconnu grâce à ses expérimentations menées sur le vivant, promoteur auprès du public des inventaires participatifs de la flore et des référents de la biodiversité, le Jardin botanique de Nantes s'associe également aux différents événements culturels et intègre le dispositif global de mise en tourisme de la ville et de sa région, montrant ainsi qu'il s'inscrit pleinement au cœur du développement des politiques territoriales locales.

\section{INDEX}

Mots-clés : jardin botanique, Nantes, politique publique

\section{AUTEUR}

\section{ROMARIC PERROCHEAU}

directeur du Jardin botanique de Nantes

romaric.perrocheau@mairie-nantes.fr 